Objectives: The aim of this study is to evaluate the efficacy and the safety of $\mathrm{HCQ}$ as co-treatment in the standard therapy of SLE.

Methods: SLE patients $(n=30)$ under the maintenance therapy were enrolled in this study. Dose of PSL, titer of anti-DNA antibody, WBC count, serum complement and SLE disease activity index (SLEDAI) were examined retrospectively at 0 and 12 months after administration of $\mathrm{HCQ}$.

Results: Baseline patient-characteristics are as follows (mean \pm S.E); the age of patients was $54.4 \pm 3.2$ years old, 21 patients $(70 \%)$ were female, the disease duration was $108.5 \pm 25.2$ months, SLEDAI was $4.0 \pm 0.9$, the dose of PSL was $10.3 \pm 1.7 \mathrm{mg} /$ day, the titer of anti-DNA antibody was $7.3 \pm 1.8 \mathrm{IU} / \mathrm{ml}$, C3 was $85 \pm 4.3 \mathrm{mg} / \mathrm{dl}$ and $\mathrm{C} 4$ was $18 \pm 1.6 \mathrm{mg} / \mathrm{dl}$.

The mean dose of PSL was reduced with statistically significance (pre-administration of $\mathrm{HCQ}: 10.3 \pm 1.7 \mathrm{mg} /$ day, 24 months after administration of $\mathrm{HCQ}: 2.2 \pm 0.3 \mathrm{mg} /$ day, $p<0.0001)$. Furthermore, in this observation period, 6 patients could achieve the cessation of PSL.

SLEDAI was also significantly reduced $(4.0 \pm 0.9$ vs $1.0 \pm 0.3, p<0.01)$.

There was no statistical significance between before treatment by HCQ and after treatment in the titer of anti-DNA antibody $(7.3 \pm 1.8$ vs $2.8 \pm 1.6 \mathrm{IU} / \mathrm{ml}, p=0.06)$, WBC count $(6208 \pm 4.9$ vs $5096 \pm 3.3 / \mu \mathrm{l}, p=0.06)$ and serum complement level (C3 $85 \pm 4.3 \mathrm{mg} / \mathrm{dl}$ vs $89 \pm 4.0 \mathrm{mg} / \mathrm{dl}, p=0.52$, C4 $18 \pm 1.6 \mathrm{mg} / \mathrm{dl}$ vs $19 \pm 1.4 \mathrm{mg} / \mathrm{dl}$, $p=0.45)$. Relapse of SLE was clarified in only one patient.

As for adverse events (AEs), Severe bacterial infection $(n=4)$, herpes zoster $(n=1)$ and patellar tendon rupture $(n=1)$ were revealed. All cases of the AEs were fully recovered. Conclusion: Our study suggested that co-treatment with HCQ on standard SLE therapy could be enable to prevent the flare of SLE and reduce the dose of PSL with statistical significance. In some cases, we could achieve the cessation of PSL treatment References: None.

Disclosure of Interests: None declared

DOI: 10.1136/annrheumdis-2020-eular.5277

\section{AB0382 $\quad$ A RITUXIMAB AND BELIMUMAB COMBINATION THERAPY IN SLE PATIENTS.}

A. Mesnyankina ${ }^{1}$, S. Solovyev ${ }^{1}$, E. Aseeva ${ }^{1}$, N. Nikishina ${ }^{1} .{ }^{1}$ VA Nasonova Research Institute of Rheumatology, Moscow, Russian Federation

Background: Various mechanisms of action of RTM and BLM, in particular their interaction with defined subpopulations of B cells, can contribute to more effective suppression of autoreactive $B$ cells and achieve a therapeutic effect.

Objectives: To assess the efficacy of a rituximab and belimumab combination therapy in pts with active SLE.

Methods: The study included 10 SLE pts $(1 \mathrm{M} / 9 \mathrm{~F})$ with high (SLEDAI2K $\geq 10-8 \mathrm{pts}$.) and moderate (SLEDAI2K<10-2pts.) disease activity; out of them 2 patients had lupus nephritis, 2- vasculitis. 1 pts both (nephritis and vasculitis), and remaining 5 had predominantly mucocutaneous and articular manifestations of SLE. The dose of oral GCs at baseline did not exceed $20 \mathrm{mg} /$ day in 9 pts, two pts were treated with prednisone $5 \mathrm{mg} /$ day and only 1 received $60 \mathrm{mg}$. Rituximab (RTM) was administered at $500-2000 \mathrm{mg}$, with subsequent adding of Belimumab (BLM) 1-6 months later at a standard dosing regimen $10 \mathrm{mg} / \mathrm{kg}$ once a month. CD19+ B- lymphocytes counts were obtained before initiation RTM (0), and subsequently after $3(\mathrm{~N}=10)$, $6(\mathrm{~N}=10), 9(\mathrm{~N}=7)$, and 12month $(\mathrm{N}=7)$. Depletion of CD19+ B- lymphocytes after RTM was assessed as the decrease of $B$-cell counts $<0,0110^{*} 9 / /$, where $010^{*} 9 / /$ was categorized as complete depletion, from 0,001 to $0,0110^{\star} 9 / \mathrm{l}$ - partial depletion, and $>0,01110^{*} 9 / l-$ absence of depletion. The comparison group included $20 \mathrm{pts}$ receiving a sing $=\mathrm{b} 500-2000 \mathrm{mg}$ dose of $\mathrm{RTM}$ with high (SLEDAI2K $\geq 10-16 \mathrm{pts}$.) and moderate (SLEDAI2K<10- 4pts.) disease activity (SLEDAI Me 14[10;16])

Results: 6 pts demonstrated the decrease in clinical and laboratory SLE activity, starting from $3 \mathrm{mo}$ of follow-up, and by the 6 th month the decrease in the activity of the disease was observed in 9 patients (SLEDAI-2K 0 mo-Me 12[10;16], 3mo-Me 8[6;10], 6mo-Me 4[2;6], 9mo-Me 6[4;10], 12mo-Me 2[2;6]) with RTM + BLM combination therapy. The oral GCs dose was reduced to 6,9 $[5 ; 10] \mathrm{mg} /$ day by $6 \mathrm{mo}$. One patient managed to completely eliminate glucocorticoids; he continued to receive cytostatic therapy (mycophenolate mofetil). None of the patient required prednisone dose escalation during follow-up. There were no cases of severe infection. The damage index did not increase by 6 and $12 \mathrm{mo}$. The combination therapy reduced the absolute counts of CD19+. B-cells. RTM therapy resulted in complete depletion in 3 pts, in partial depletion - in 4. Addition of BLM resulted in slowing down of CD19+ B-cell repopulation (Fig.1) (0moMe $0,11 \times 10^{9} /[0,08 ; 0,5], 12 \mathrm{mo}-\mathrm{Me} 0,01 \times 10^{9} /[[0,01 ; 0,03])$ vs pts receiving RTM

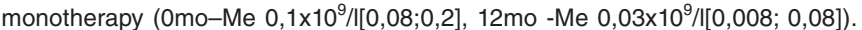
RTM and BLM combination failure, as well as failure of standard GCS and cytostatic based therapy, was documented in one patient with cutaneous, articular and hematological SLE.

Conclusion: Combination therapy allows to gain control over disease activity in short time, due to the effect of RTM, while added BLM provides further prolongation of the effect achieved, minimizing the risk of exacerbation. This combination may be used as a method of choice in pts with severe SLE involving vital organs, and in persistent cutaneous-articular disease and high immunological activity. In these patients there were no signs of infection.

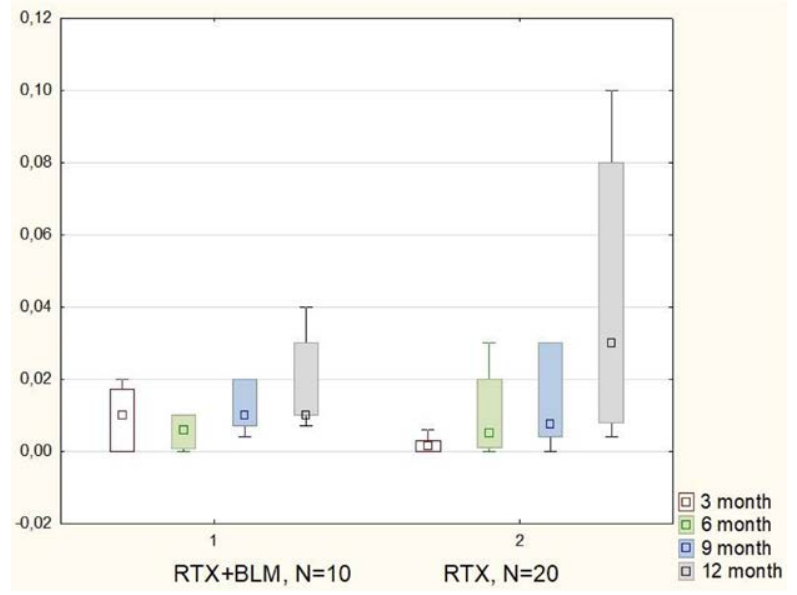

Figure 1.

Disclosure of Interests: None declared

DOI: 10.1136/annrheumdis-2020-eular.4217

\section{AB0383 $\quad$ EXTREME FATIGUE IN PATIENTS WITH SYSTEMIC LUPUS ERYTHEMATOSUS AND NEUROPSYCHIATRIC SYMPTOMS}

R. Monahan ${ }^{1}$, R. Fronczek ${ }^{2}$, J. Eikenboom ${ }^{3}$, H. Middelkoop ${ }^{2}$, L. J. J. Beaart- van de Voorde $^{1}$, G. Terwindt ${ }^{2}$, N. Van der Wee ${ }^{4}$, T. Huizinga ${ }^{1}$, M. Kloppenburg ${ }^{1}$, G. M. Steup-Beekman ${ }^{1} .{ }^{1}$ Leiden University Medical Center, Rheumatology, Leiden, Netherlands; ${ }^{2}$ Leiden University Medical Center, Neurology, Leiden, Netherlands; ${ }^{3}$ Leiden University Medical Center, Internal Medicine, Division of Thrombosis and Hemostasis, Leiden, Netherlands; ${ }^{4}$ Leiden University Medical Center, Psychiatry, Leiden, Netherlands

Background: Fatigue is commonly described in chronic illnesses, especially auto-immune disorders such as systemic lupus erythematosus (SLE).

Objectives: We aim to study the prevalence of fatigue in SLE patients with NP symptoms and compare fatigue in SLE patients with NP symptoms attributed to major organ involvement due to SLE (NPSLE) with SLE patients with NP symptoms not caused by major nervous system involvement (non-NPSLE).

Methods: All patients visiting the tertiary referral center for NPSLE in the LUMC between 2007-2019 with the clinical diagnosis of SLE and age $>18$ years that signed informed consent were included in this study. Patients underwent a standardized multidisciplinary assessment, including two questionnaires: SF-36 (2007-2019) and multidimensional fatigue index (MFI, 2011-2019). Patients were classified as NPSLE in this study if NP symptoms were attributed to SLE and immunosuppressive or anticoagulant therapy was initiated, otherwise patients were classified as non-NPSLE. The vitality (VT) domain of the SF-36 domain was used to assess fatigue, which generates a score from 0-100, 100 representing the complete absence of fatigue. Patients with a score more than 1 standard deviation (SD) removed from age-related controls of the Dutch general population were classified as fatigued; patients more than 2 SD removed were classified as extremely fatigued ${ }^{1}$. The MFI was also used, which consists of 5 subdomain scores between $0-20$, leading to a total score between $0-100,100$ representing the most extreme fatigue. All scores are presented as mean and standard deviation. Results: 373 patients fulfilled the inclusion criteria and SF-36 questionnaires of 328 patients were available (88\%). The majority of these patients was female $(87 \%)$ and 98 were classified as NPSLE (30\%). In NPSLE patients, average age was 41 \pm 13 years and in non-NPSLE the average age was $45 \pm 14$ years. The average score of the SF-36 vitality domain was $36.0 \pm 20.7$ in NPSLE vs $33.9 \pm 18.8$. in nonNPSLE. Overall, $73.5 \%$ of the patients were fatigued and $46.9 \%$ extremely fatigued in NPSLE vs $77.8 \%$ fatigued and $45.7 \%$ extremely fatigued in non-NPSLE. The MFI questionnaire and VAS score were available for 222 patients, of which 65 patients were classified as NPSLE (29.3\%). Table 1 depicts the scores of NPSLE and non-NPSLE patients on the MFI subdomains and the VAS score.

Table 1. Fatigue in NPSLE and non-NPSLE patients $(\mathrm{N}=\mathbf{2 2 2})$

\begin{tabular}{lcc}
\hline & NPSLE (N = 65) & Non-NPSLE (N = 157) \\
\hline MFI (mean, sd) & & \\
General Fatigue & $10.8(1.8)$ & $11.1(1.5)$ \\
Physical Fatigue & $11.4(2.4)$ & $12.3(1.9)$ \\
Reduced Activity & $9.6(2.9)$ & $10.7(2.2)$ \\
Reduced Motivation & $10.7(2.6)$ & $11.1(1.9)$ \\
Mental Fatigue & $9.5(3.0)$ & $9.8(2.7)$ \\
Total score & $51.8(9.9)$ & $54.9(6.9)$ \\
SF-36 Vitality (mean, sd) & $35(20.7)$ & $32.7(18.2)$
\end{tabular}

